\title{
Prognostic value of matrix metalloproteinase-9 expression in oral squamous cell carcinoma and its association with angiogenesis
}

\author{
Azadeh Andisheh-Tadbir ${ }^{1}$, Maryam Mardani ${ }^{2}$, Sara Pourshahidi ${ }^{3}$, Kamran Nezarati ${ }^{4}$, Parisa Bahadori ${ }^{5}$ \\ ${ }^{1}$ Associate Professor, Prevention of Oral and Dental Disease Research Center, School of Dentistry, Shiraz University of Medical \\ Sciences, Shiraz, Iran \\ ${ }^{2}$ Assistant Professor, Department of Oral Medicine, School of Dentistry, Shiraz University of Medical Sciences, Shiraz, Iran \\ ${ }^{3}$ Assistant Professor, Department of Oral Medicine, School of Dentistry, Tehran University of Medical Sciences, Tehran, Iran \\ ${ }^{4}$ Undergraduate Student, School of Dentistry, Shiraz University of Medical Sciences, Shiraz, Iran \\ ${ }^{5}$ Undergraduate Student, School of Dentistry, International Branch, Shiraz University of Medical Sciences, Shiraz, Iran
}

Correspondence:

Department of Oral Medicine

School of Dentistry

Tehran University of Medical Sciences

Tehran, Iran

andisheh202003@yahoo.com

Andisheh-Tadbir A, Mardani M, Pourshahidi S, Nezarati K, Bahadori P. Prognostic value of matrix metalloproteinase-9 expression in oral squamous cell carcinoma and its association with angiogenesis. J Clin Exp Dent. 2016;8(2):e130-5.

http://www.medicinaoral.com/odo/volumenes/v8i2/jcedv8i2p130.pdf

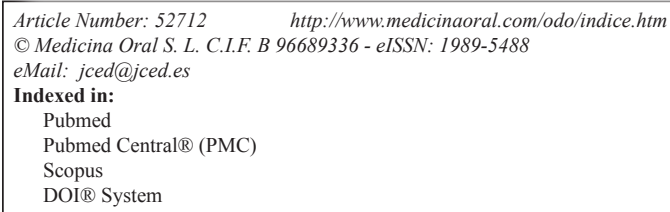

\begin{abstract}
Background: Breakdown of extracellular matrix $(\mathrm{ECM})$ is one of the important hallmarks of cancer progression which facilitates the invasion of tumoral cells to the surrounding tissue. Matrix metalloproteinases (MMPs) can degrade various components of the ECM and basement membrane. The aim of this study was to determine the role of matrix metalloproteinases-9 protein in the biologic behavior of oral squamous cell carcinoma (OSCC) and its relation with tumor angiogenesis.

Material and Methods: In this study 42 OSCC and 15 normal epithelium were reviewed by immunohistochemical staining for matrix metalloproteinases-9 and CD105.

Results: Matrix metalloproteinases-9 expression was detected in 32 OSCC specimens (76.1\%), with 28 specimens $(66.6 \%)$ showing moderate or strong expression. We observed that the expression level of matrix metalloproteinases-9 was positively correlated with the status of lymph node metastasis (N0vs. N1) $(P=0.00)$, and clinical stage (I-II vs. III-IV) in OSCC patients. Microvessel density in intratumoral tissue has an association with lymph node metastasis and advanced clinical stage $(P=0.003$ and $p=0.01$, respectively). We observed that tumors with matrix metalloproteinases- 9 overexpression had a higher microvessel density counts compared with tumors with absent or focal immunostaining ( $16.2 \pm 5.6$ vs $10.3 \pm 3.5$ respectively, $P=0.03$ ).

Conclusions: In conclusion present results demonstrate the marked expression of matrix metalloproteinases-9 and CD105 in OSCC and suggest that the expression of these markers is associated with tumor progression and could offer additional information about the aggressiveness of OSCC. In addition a significant relationship was noted between microvessel density count and expression of matrix metalloproteinases-9 which suggest that MMP9 expression may be closely related to tumor angiogenesis.
\end{abstract}

Key words: Matrix metalloproteinases-9, CD105, squamous cell carcinoma, immunohistochemistry. 


\section{Introduction}

Squamous cell carcinoma (SCC) accounts for approximately $95 \%$ of oral malignant neoplasms and $38 \%$ of all malignant head and neck tumors (1). Different factors such as the degree of tumor differentiation, the proliferative activity of the tumor, and the invasion and metastatic potential affect the prognosis of SCC (2). Invasion and metastasis are multi-step processes that include basement membrane and ECM degradation, changes in cell adhesiveness, motility of tumor cells, and angiogenesis (3).

The investigation of the factors impacting these processes is important to understand tumor behavior and for the development of anticancer therapies.

Angiogenesis, which is critical for growth of tumor and metastasis, depends on the angiogenic factors which are produced by normal and tumoral cells. It implicates several pathways comprising the production of angiogenic factors, endothelial cells activation, and destruction of capillary membrane and migration of endothelial cells (4).

Increased vascularity improves the growth of primary neoplasms and increases the chance for hematogenous metastasis (5).

Microvessel density (MVD) is a quantitative method for analysis of angiogenesis which can be investigated by using various molecules, such as CD31, CD34, and CD105 (6,7).

CD105 is a homodimeric cell membrane glycoprotein and is a component of TGF- $\beta$ receptor complex. This marker is an indicator of endothelial cell proliferation and is up-regulated during angiogenesis (8). In addition, expression of CD105 is one of the prominent characteristics of newly formed blood vessels and its expression is negative or insignificant in previously formed blood vessels, endothelium of the vessels of normal tissues and endothelial cells of lymphatic vessels (9).

Breakdown of ECM is one of the important hallmarks of cancer progression which facilitates the invasion of tumoral cells to the surrounding tissue. Matrix metalloproteinases (MMPs) are zinc dependent endopeptidases that can degrade various components of the ECM and basement membrane (BM) (10).

To date, at least 24 different MMP genes have been recognized in humans. MMPs are classified according to their substrate specificities collagenenases, gelatinases, stromelysins and matrilysins (11). Two different soluble gelatinase have been identified: gelatinase A, $72 \mathrm{KDa}$ (MMP-2), and gelatinase B, 92KDa (MMP9) (12).

MMP9 has an important role in breakdown of ECM in normal physiological processes, such as embryonic development and tissue remodeling, as well as in the pathologic processes, such as a tumor metastasis (13).

MMP9 is secreted in a latent form and once activated, is able to degrade collagen in the ECM, which increases the metastasis of tumor cells (14).
The aim of the present study was to evaluate the immunohistochemical expression of MMP9 and CD105 in OSCC in order to determine the presence or absence of a correlation between the expression of these proteins and the clinicopathologic features.

\section{Material and Methods}

-Materials

In this cross-sectional study, the specimen from 42 patients with oral SCC ( 28 males and 14 females) with a mean age 54.47 (range35-81) from the archives of Pathology Department of Shiraz University of Medical Sciences (2008-2012) were studied. The control group was consisted of 15 cases of normal oral epithelium. This study was approved by the Ethics Committee of the Shiraz University of Medical Sciences.

-IHC staining and analysis

Firstly, Hematoxillin and Eosin slides of available blocks were reviewed and then cases with definite diagnosis and adequate cellular tissue were selected for immunohistochemical staining (IHC). IHC staining was performed by using EnvsionLabled Peroxides System (DAKO, Carpentaria, CA, USA). All the samples were fixed in $10 \%$ buffered formalin and were embedded in paraffin. Sections with $4 \mu$-thickness were prepared, deparaffinized in xylene, rehydrated in graded alcohol and were washed with distilled water. Antigen retrieval was performed by using DAKO cytomation target retrieval solution with $\mathrm{PH}=9$, for 20 minutes. Internal peroxidase activity was inhibited by $3 \% \mathrm{H}_{2} \mathrm{O}_{2}$.

Tissue sections were then incubated for 30 minutes with the anti-CD105 monoclonal antibody (mouse, Dako Corporation, Denmark) at a 1/10 dilution and with the anti-MMP-9 antibody (Santa Cruz Biotechnology Inc., Sc-19993) at a 1/50 dilution. Brown cytoplasmic staining for CD105 and MMP- 9 was considered as positive. Omission of primary antibody was considered as negative control, while liver tissue was used as positive control for CD105 and human colon adenocarcinoma for MMP-9.

For determining MVD hot-spot areas for CD105 expression in discrete blood vessels were initially identified by scanning the entire tumor of low power (X40) and the number of CD105 highlighted vessels in 10 of these areas was then counted in high power magnification (X400).

For analysis MMP-9 expression five microscopic fields in tumour tissues (original magnification $400 \times$ ) were randomly selected and the percentage of positive cells was calculated. The percentage of positive cells was divided into four groups as follows: $0-25 \%$,negative expression; $25-50 \%$, weak expression; $50-75 \%$,moderate expression; and $75-100 \%$, strong expression. For statistical analysis, the patients were classified into two groups: 'low expression' included those with negative 
or weak expression and 'high expression' included those with moderate or strong expression.

-Statistical analysis

T-test, Independent samples t- test, paired T-test, Pearson correlation, and chi square test were used to compare the results between the two groups and the relation with clinicopathologic features. We used software SPSS15 to statically analyze the data. A $P$-value $<0.05$ was considered significant in all the statistical analyses.

\section{Results}

The clinicopathologic features of the patients included in this study are shown in tablel. The mean age of the investigated patients was 54.4 \pm 12.44 (range 35-81). Gender of the patients with OSCC included 28 males $(66.7 \%)$ and 14 females (33.3\%).

-Immunohistochemical analysis of MMP-9 expression Positive expression of MMP-9 was mainly observed in the cytoplasm of stromal cells and proliferating epithelial cells as brownish granules under $400 \times$ (Figs. 1,2).

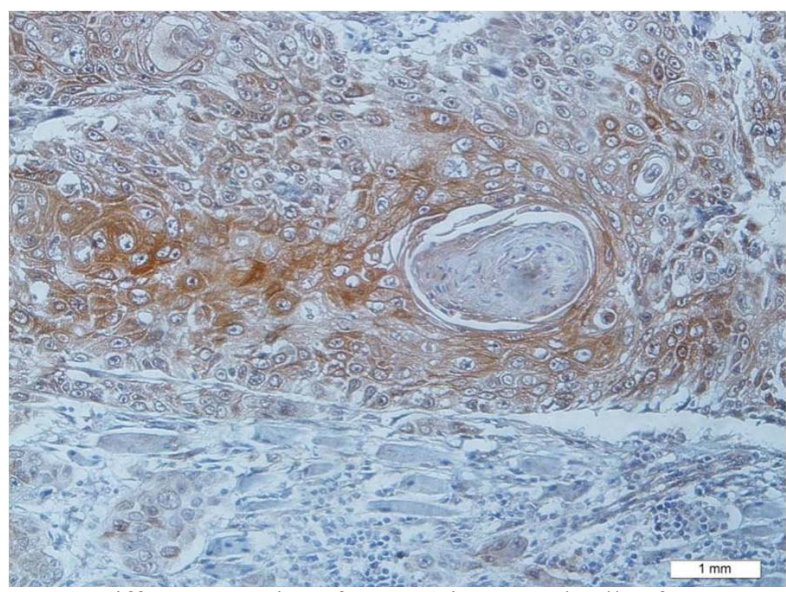

Fig. 1. Diffuse expression of MMP-9 in tumoral cells of squamous cell carcinoma $(\times 200)$.

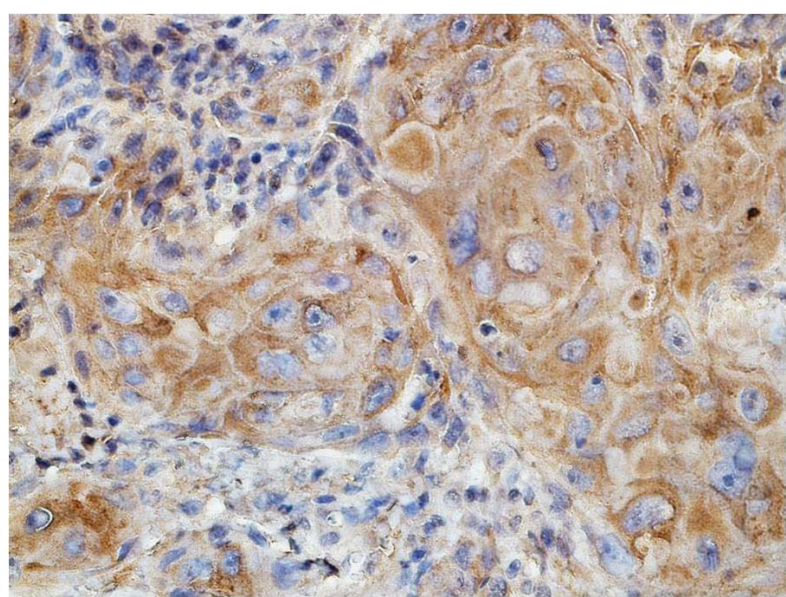

Fig. 2. Diffuse cytoplasmic expression of MMP-9 in tumoral and stromal cells of squamous cell carcinoma $(\times 400)$.
The expression of MMP-9 was not seen in normal oral epithelium. MMP-9 expression was detected in 32 OSCC specimens (76.1\%), with 28 specimens $(66.6 \%)$ showing moderate or strong expression.

-Relationship between clinicopathological characteristics and MMP9 expression in OSCC patients

The relationships between clinicopathological characteristics and MMP9 expression levels in individuals with OSCC are summarized in table 1 . We did not find a significant association of MMP9 expression levels with patient's age, sex, and tumor size ( $\mathrm{T}$ classification). However, we observed that the expression level of MMP9 was positively correlated with the status of lymph node metastasis (N classification) (N0 vs. N1) $(P$ $=0.00)$, and clinical stage (I-II vs. III-IV) $(P=0.00)$ in OSCC patients (Table 1).

-MVD assessment

The mean CD105-MVD value was significantly higher in tumoral tissue $(20.02 \pm 8.03)$ when compared to normal tissues (8.67 \pm 1.75$)$ (Fig. 3). MVD in intratumoral tissue had an association with lymph node metastasis and advanced clinical stage ( $P=0.003$ and $p=0.01$, respectively). There was no relation between the CD105MVD value and tumor size in intratumoral area $(P$ value $=0.05$ ). Statistical analysis showed a negative association between grade of the tumor in intratumoral region $(P$-value $=0.03)$. We did not observe any correlation between CD105-MVD with age and sex $(P=0.4$ and $p=0.7$ respectively). The correlation of the CD105-MVD value with clinicopathologic data is shown in table 1 .

We analyzed the correlation between MVD and MMP9 expression and we observed that tumors with MMP-9 overexpression had a higher MVD counts compared with tumors with absent or focal immunostaining (16.2 \pm 5.6 vs $10.3 \pm 3.5$, respectively $P=0.03$ ).

\section{Discussion}

The expression and activation of proteolytic enzymes, which are involved in the ECM breakdown, is necessary for the invasion and spread of tumoral cells to the distant sites (15).

MMPs are a family of proteolytic enzymes with the ability of ECM degradation that has a critical role in several stages of tumor progression, consisting invasion, angiogenesis, and metastasis (16).

Among the various techniques that can be used to evaluate MMP expression in the tumoral tissue, immunohistochemistry demonstrates advantages; first because it permits direct correlation with morphology; and, second it can be used on paraffin embedded tissue.Therefore, it is useful for routine assessment of MMPs in diagnostic practice. Moreover, the immunohistochemicals studies are crucial since mRNA may not be translated, while protein expression has more functional relevance (17). However, it has been proposed that immunohistoche- 
Table 1. Relationship between MMP-9 expression, MVD and clinicopathologic parameters in patients with oral squamous cell carcinoma.

\begin{tabular}{|c|c|c|c|c|c|c|}
\hline & \multirow{2}{*}{$\begin{array}{l}\text { Frequency } \\
(\mathrm{N})(\%)\end{array}$} & \multirow{2}{*}{$\begin{array}{c}\text { Intratumoral } \\
\text { MVD }\end{array}$} & \multirow[t]{2}{*}{$P$ value } & \multicolumn{3}{|c|}{ MMP-9 expression } \\
\hline & & & & $($ Low $)(\%)$ & (high) $(\%)$ & $P$ value \\
\hline $\begin{array}{l}\text { SEX } \\
\text { Male } \\
\text { Female }\end{array}$ & $\begin{array}{l}28(66.6) \\
14(33.4)\end{array}$ & $\begin{array}{l}15.2 \pm 5.5 \\
15.7 \pm 4.3\end{array}$ & 0.7 & $\begin{array}{c}8(28.6) \\
7(50)\end{array}$ & $\begin{array}{c}20(71.4) \\
7(50)\end{array}$ & 0.18 \\
\hline $\begin{array}{l}\text { Tumor Size } \\
\mathrm{T} 1+\mathrm{T} 2 \\
\mathrm{~T} 3+\mathrm{T} 4\end{array}$ & $\begin{array}{l}32(76.1) \\
10(23.9)\end{array}$ & $\begin{array}{l}14.8 \pm 4.9 \\
16.8 \pm 5.9\end{array}$ & 0.05 & $\begin{array}{c}13(40.6) \\
1(10)\end{array}$ & $\begin{array}{c}19(59.4) \\
9(90)\end{array}$ & 0.1 \\
\hline $\begin{array}{l}\text { Lymph node } \\
\text { involvement } \\
\text { N0 } \\
\text { N1 }\end{array}$ & $\begin{array}{l}21(50) \\
21(50)\end{array}$ & $\begin{array}{l}13.1 \pm 2.9 \\
17.3 \pm 6.3\end{array}$ & 0.003 & $\begin{array}{c}14(66.7) \\
0(0)\end{array}$ & $\begin{array}{l}7(33.3) \\
21(100)\end{array}$ & 0.000 \\
\hline $\begin{array}{l}\text { Grade } \\
\text { G1 } \\
\text { G2 } \\
\text { G3 }\end{array}$ & $\begin{array}{c}27(64.3) \\
12(28.5) \\
3(7.2)\end{array}$ & $\begin{array}{l}16.6 \pm 5.8 \\
12.7 \pm 2.0 \\
13.0 \pm 1.0\end{array}$ & 0.03 & $\begin{array}{l}11(40.7) \\
2(16.7) \\
1(33.3)\end{array}$ & $\begin{array}{l}16(59.3) \\
10(83.3) \\
2(66.7)\end{array}$ & 0.3 \\
\hline $\begin{array}{l}\text { Stage } \\
\text { I + II } \\
\text { III + IV }\end{array}$ & $\begin{array}{l}19(45.2) \\
23(54.8)\end{array}$ & $\begin{array}{l}12.9 \pm 3.8 \\
17.2 \pm 5.7\end{array}$ & 0.01 & $\begin{array}{c}13(68.4) \\
1(4.3)\end{array}$ & $\begin{array}{c}6(31.6) \\
22(95.7)\end{array}$ & 0.000 \\
\hline
\end{tabular}

MVD: Microvessel density.

MMP: Matrix metalloproteinase.

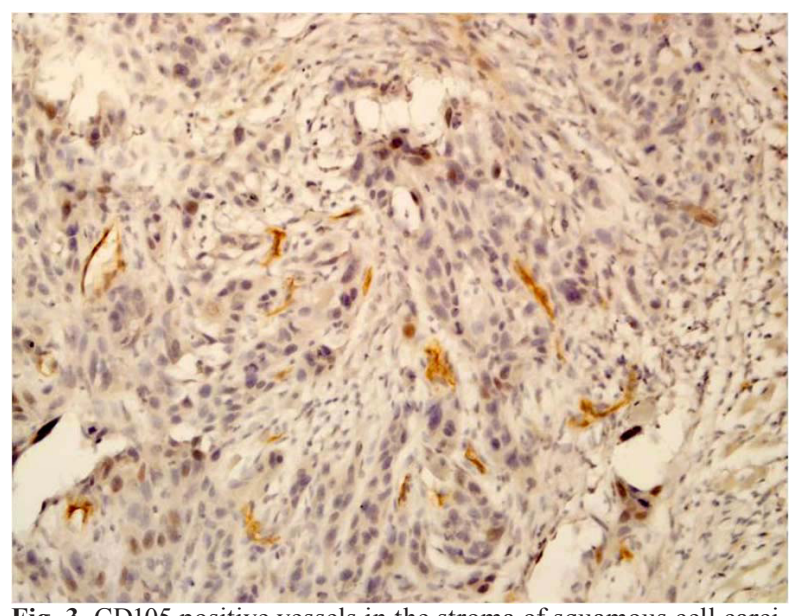

Fig. 3. CD105 positive vessels in the stroma of squamous cell carcinoma $(\times 200)$.

mistry cannot separate latent MMPs and active forms, while other techniques such as zymography can distinguish between the latent and active morphology (18).

Concerning MMP9, in the present study we found that $76.1 \%$ of OSCCs exhibited MMP9 immunoreactivity in tumor tissues and no expression is seen in normal tissues. These findings suggest that this molecule play an effective role in the development of these tumors.

In our study, the expression of MMP9 was found largely in tumor cells but also in the adjacent stromal and inflammatory cells. In view of this finding, our study supports previous findings and provides further evidence that both tumoral and stromal cells can produce different members of MMPs in tumor tissues. Indeed, it is possible that dynamic host-tumor interactions regulate MMPs level and affect the progression of human tumors (15). Several studies demonstrated a relationship between the MMP9 expression and tumor aggressiveness in OSCC a fact that made this factor as a valuable marker for prognostic and therapeutic purposes (19). Werner et al. (20) showed that the overexpression of MMP9 is correlated with an invasive phenotype and metastatic potential of tumor cells.

In this study, MMP9 overexpression was significantly correlated with lymph node metastasis and advanced clinical stage. This finding reveals the significance of this enzyme in the aggressive phenotype of OSCCs through the destruction of ECM components, especially collagen IV (21).

Angiogenesis has been proposed to have a prognostic factor in tumors because it is necessary for tumor growth (5). In the current study we analyzed specimens from 42 patients diagnosed with OSCC and 15 normal specimens as the control group. Our study proved that CD105MVD was significantly higher in tumoral tissue compared with normal tissue which was in line with previous studies $(22,23)$. 
These results have verified that CD105 is more expressed in tumor tissues and may have a major role in the tumor. We also observed a positive relation between CD105 expression and lymph node metastasis. This finding was compatible with previous investigations (2426) and suggested that the marker could be helpful in predicting the possibility of metastasis.

Angiogenesis is prerequisite for the growth, proliferation and metastasis of cancer cells. Therefore, these processes can be inhibited through inhibiting angiogenesis (27). At present there are several antiangiogenic agents that affect the neovascularization pathway, but none of them has a predictive marker (28). High MVD alone is not a predictive marker for anti-angiogenic agents since antiangiogenic agents target new vessels in tumors only. As CD105 expression is more specific for areas of neovascularization, CD105 has the potential to be a predictive marker for anti-angiogenic agents (27).

MMPs can improve tumor progression through affecting angiogenesis, because these enzymes degrade the ECM and offer a permissive microenvironment for the growth of new blood vessels. In this study, we observed that OSCC tumors with MMP9 overexpression had significantly higher MVD. Similar results were obtained previously by Franchi et al. (15) and Riedel et al. (29) in head and neck SCCs. A weak association also was found between MMP9 expression and tumor angiogenesis in breast carcinoma (30). Thus, MMP9 may be an important modulator of angiogenesis in OSCC, but further investigation is needed to clarify its specific mechanism of action.

In conclusion present results demonstrate the marked expression of MMP9 and CD105 in OSCC and suggest that the expression of these markers is associated with tumor progression and could offer additional information about the aggressiveness of OSCC. In addition a significant relationship was noted between MVD count and expression of MMP9 which suggest that MMP9 expression may be closely related to tumor angiogenesis.

\section{References}

1. Scully C. Oral squamous cell carcinoma; from an hypothesis about a virus, to concern about possible sexual transmission. Oral Oncol. 2002;38:227-34.

2. Kim SH, Cho NH, Kim K, Lee JS, Koo BS, Kim JH, et al. Correlations of oral tongue cancer invasion with matrix metalloproteinases (MMPs) and vascular endothelial growth factor (VEGF) expression. J Surg Oncol. 2006;93:330-7.

3. Lopes FF, da Costa Miguel MC, Pereira AL, da Cruz MC, de Almeida Freitas R, Pinto LP, et al. Changes in immunoexpression of Ecadherin and beta-catenin in oral squamous cell carcinoma with and without nodal metastasis. Ann Diagn Pathol. 2009;13:22-9.

4. Folkman J. What is the evidence that tumors are angiogenesis dependent? J Natl Cancer Inst. 1990;82:4-6.

5. El-Shahat M, Lotfy M, Fahmy L, Abouel-Nour MF, El-Kenawy Ael M. Prognostic value of microvessel density, matrix metalloproteinase-9 and p53 protein expression in esophageal cancer. J Egypt Natl Canc Inst. 2004;16:224-30.

6. Kademani D, Lewis JT, Lamb DH, Rallis DJ, Harrington JR. An- giogenesis and CD34 expression as a predictor of recurrence in oral squamous cell carcinoma. J Oral Maxillofac Surg. 2009;67:1800-5.

7. Fox SB, Harris AL. Histological quantitation of tumour angiogenesis. Apmis. 2004;112:413-30.

8. Dallas NA, Samuel S, Xia L, Fan F, Gray MJ, Lim SJ, et al. Endoglin (CD105): a marker of tumor vasculature and potential target for therapy. Clin Cancer Res. 2008;14:1931-7.

9. Nikiteas NI, Tzanakis N, Theodoropoulos G, Atsaves V, Christoni $\mathrm{Z}$, Karakitsos P, et al. Vascular endothelial growth factor and endoglin (CD-105) in gastric cancer. Gastric Cancer. 2007;10:12-7.

10. Vilen ST, Salo T, Sorsa T, Nyberg P. Fluctuating roles of matrix metalloproteinase-9 in oral squamous cell carcinoma. Scientific World Journal. 2013;2013:920595.

11. Hadler-Olsen E, Fadnes B, Sylte I, Uhlin-Hansen L, Winberg JO. Regulation of matrix metalloproteinase activity in health and disease. Febs j. 2011;278:28-45.

12. Bjorklund M, Koivunen E. Gelatinase-mediated migration and invasion of cancer cells. Biochim Biophys Acta. 2005;1755:37-69.

13. Fridman R, Toth M, Pena D, Mobashery S. Activation of progelatinase B (MMP-9) by gelatinase A (MMP-2). Cancer Res. 1995;55:254855 .

14. Backstrom JR, Tokes ZA. The $84-\mathrm{kDa}$ form of human matrix metalloproteinase-9 degrades substance $\mathrm{P}$ and gelatin. J Neurochem. 1995;64:1312-8.

15. Franchi A, Santucci M, Masini E, Sardi I, Paglierani M, Gallo O. Expression of matrix metalloproteinase 1, matrix metalloproteinase 2, and matrix metalloproteinase 9 in carcinoma of the head and neck. Cancer. 2002;95:1902-10.

16. Ohashi K, Nemoto T, Nakamura K, Nemori R. Increased expression of matrix metalloproteinase 7 and 9 and membrane type 1-matrix metalloproteinase in esophageal squamous cell carcinomas. Cancer. 2000;88:2201-9.

17. Curran S, Murray GI. Matrix metalloproteinases in tumour invasion and metastasis. J Pathol. 1999;189:300-8.

18. Baker EA, Bergin FG, Leaper DJ. Matrix metalloproteinases, their tissue inhibitors and colorectal cancer staging. Br J Surg. 2000;87:1215-21.

19. Katayama A, Bandoh N, Kishibe K, Takahara M, Ogino T, Nonaka $\mathrm{S}$, et al. Expressions of matrix metalloproteinases in early-stage oral squamous cell carcinoma as predictive indicators for tumor metastases and prognosis. Clin Cancer Res. 2004;10:634-40.

20. Werner JA, Rathcke IO, Mandic R. The role of matrix metalloproteinases in squamous cell carcinomas of the head and neck. Clin Exp Metastasis. 2002;19:275-82.

21. Engsig MT, Chen QJ, Vu TH, Pedersen AC, Therkidsen B, Lund LR, et al. Matrix metalloproteinase 9 and vascular endothelial growth factor are essential for osteoclast recruitment into developing long bones. J Cell Biol. 2000;151:879-89.

22. Eshghyar N, Mohammadi N, Rahrotaban S, Motahhary P, Vahedi Vaez SM. Endoglin (CD105) positive microvessel density and its relationship with lymph node metastasis in squamous cell carcinoma of the tongue. Arch Iran Med. 2011;14:276-80.

23. Mărgăritescu C, Pirici D, Stîngă A, Simionescu C, Raica M, Mogoantă L, et al. VEGF expression and angiogenesis in oral squamous cell carcinoma: an immunohistochemical and morphometric study. Clin Exp Med. 2010;10:209-14.

24. Miyahara M, Tanuma J, Sugihara K, Semba I. Tumor lymphangiogenesis correlates with lymph node metastasis and clinicopathologic parameters in oral squamous cell carcinoma. Cancer. 2007;110:128794.

25. Chien CY, Su CY, Hwang CF, Chuang HC, Hsiao YC, Wu SL, et al. Clinicopathologic significance of CD105 expression in squamous cell carcinoma of the hypopharynx. Head Neck. 2006;28:441-6.

26. Chien CY, Su CY, Hwang CF, Chuang HC, Chen CM, Huang CC. High expressions of CD105 and VEGF in early oral cancer predict potential cervical metastasis. J Surg Oncol. 2006;94:413-7.

27. Rau KM, Huang CC, Chiu TJ, Chen YY, Lu CC, Liu CT, et al. Neovascularization evaluated by CD105 correlates well with prognostic factors in breast cancers. Exp Ther Med. 2012;4:231-6. 
28. Niers TM, Richel DJ, Meijers JC, Schlingemann RO. Vascular endothelial growth factor in the circulation in cancer patients may not be a relevant biomarker. PLoS One. 2011;6:e19873.

29. Riedel F, Gotte K, Schwalb J, Bergler W, Hormann K. Expression of 92-kDa type IV collagenase correlates with angiogenic markers and poor survival in head and neck squamous cell carcinoma. Int J Oncol. 2000;17:1099-105.

30. Kurizaki T, Toi M, Tominaga T. Relationship between matrix metalloproteinase expression and tumor angiogenesis in human breast carcinoma. Oncol Rep. 1998;5:673-7.

\section{Acknowledgements}

The authors thank the Vice-Chancellery of Shiraz University of Medical Sciences for supporting this research (Grant \#93-7329).This manuscript is based on the thesis of Kamran Nezarati for partial fulfillment of DDS degree. The authors are grateful to Dr. M. Salehi of the Dental Research Development Center of the Dental School, for the statistical analysis, and Dr. Sh. Hamedani (DDS, MSc) for his editorial assistance.

\section{Conflict of Interest}

There is no conflicts of interest. 